ORIGINAL ARTICLE

\title{
Mycobacterium Tuberculosis and Nontuberculous Mycobacteria Isolates from Presumptive Pulmonary Tuberculosis Patients Attending A Tertiary Hospital in Addis Ababa, Ethiopia
}

\author{
Daniel Kahase $^{1 *}$, Kassu Desta ${ }^{2}$, Zelalem Yaregal ${ }^{3}$, Bazezew Yenew ${ }^{3}$, Getu \\ Driba $^{3}$, Hilina Molalign ${ }^{3}$, Absra Solomon ${ }^{1}$, Fitsum Bekele ${ }^{1}$
}

OPEN ACCESS

Citation: Daniel Kahase, Kassu Desta, Zelalem Yaregal, Bazezew Yenew, Getu Driba, Hilina Molalign, Absra Solomon, Fitsum Bekele. Mycobacterium Tuberculosis and Nontuberculous Mycobacteria Isolates from Presumptive Pulmonary Tuberculosis Patients Attending St. Paul's Hospital Millennium Medical College, Addis Ababa, Ethiopia. Ethiop J Health Sci. 2021;31 (1):15. doi:http://dx.doi.org/ 10.4314/ejhs.v31i1.3

Received: August 3, 2020

Accepted: July 28, 2020

Published: August 4, 2020

Copyright: (C) 2021 Daniel Kahase, et al. This is an open access article distributed under the terms of the Creative Commons Attribution License, which permits unrestricted use, distribution, and reproduction in any medium, provided the original author and source are credited.

Funding: Addis Ababa University Competing Interests: The authors declare that this manuscript was approved by all authors in its form and that no competing interest exists.

Affiliation and Correspondence:

${ }^{1}$ Department of Medical Laboratory Sciences, College of Medicine and Health Sciences, Wolkite University, South Nation Nationality and peoples, Ethiopia

${ }^{2}$ Department of Medical Laboratory Sciences, College of Health Sciences, Addis Ababa University, Addis Ababa, Ethiopia

${ }^{3}$ National Tuberculosis Laboratory, Ethiopian Public Health Institutes, Addis Ababa, Ethiopia

*Email: danielasalfew@gmail.com

\section{ABSTRACT}

BACKGROUND: Mycobacterial infections are known to cause a public health problem globally. The burden of pulmonary disease from nontuberculous mycobacteria is reportedly on the rise in different parts of the world despite the fact that there is limited data about the disease in sub-Saharan Africa including Ethiopia. Hence, we aimed to assess the magnitude of $M$. tuberculosis and nontuberculous mycobacteria (NTM) among presumptive pulmonary tuberculosis patients attending St. Paul's hospital Medical College, Addis Ababa, Ethiopia.

METHODS: A cross-sectional study was conducted from June to September 20/2016. Morning sputum specimens were collected, processed and cultured in Lowenstein Jensen medium and BACTEC MGIT 960 media. The nontuberculous mycobacteria were further confirmed and characterized by Genotype CM/AS assays. The socio-demographic, clinical and chest $x$-ray data were collected using a structured questionnaire. The data was analyzed using SPSS version 20.

RESULTS: Out of 275 presumptive tuberculosis patients enrolled in the study, 29(10.5\%) were culture positive for Mycobacteria. Of these, 3(10.3\%) were found to be NTM and 26(89.6\%) were Mycobacterium tuberculosis complex. Of the NTM, two were unidentified and one typed as M.peregrinum. There was no coisolation of Mycobacterium tuberculosis complex and nontuberculous mycobacteria. Overall, 6(23.1\%) Mycobacterium tuberculosis complex isolates were resistant to at least one antituberculosis drug. Of these, two were multidrug resistant tuberculosis cases (7.7\%) detected from previously treated patients.

CONCLUSION: Relatively low magnitude of Mycobacterium tuberculosis complex and nontuberculous mycobacteria isolates were seen in the study area. Therefore, further study using a large sample size is needed to be done to consider nontuberculous mycobacteria infection as a differential diagnosis in presumptive pulmonary tuberculosis patients.

KEYWORDS: Nontuberculous mycobacteria, Multi drug resistant tuberculosis, Mycobacterium tuberculosis complex 


\section{INTRODUCTION}

The genus Mycobacteria includes aerobic and non-motile organisms. They have a lipid rich cell wall which is thicker than most other bacteria (1). Both Mycobacterium tuberculosis and nontuberculous mycobacteria (NTM) classified in this genus are acid fast bacilli indistinguishably and cause chronic lung infections (2,3). They also share clinical manifestation, features of hardiness, hydrophobicity, aerosolization and intracellular pathogenicity $(4,5)$. However, person-to-person transmission has not been convincingly reported in NTM pulmonary disease (6).

Non-tuberculous mycobacteria encompass all Mycobacterium species that are not members of the Mycobacterium tuberculosis complex and Mycobacterium leprae. They are free-living organisms found ubiquitously in the environment mainly in soil and water. More than 125 species of NTM are identified, approximately 60 of which are suspected or known to be pathogenic $(7,8)$. NTM can cause chronic pulmonary disease, disseminated infection, lymphadenitis, and skin and soft tissue infection in both immunocompetent and immunocompromised individuals $(7,8,9)$.

Globally, over the past three decades prevalence of pulmonary NTM disease has increased dramatically (10). These could be partially due to factors like the development of microbiological isolation and identification techniques, pathogen, host and host parasite interaction factors (11). Increasing numbers of immune compromised patients, including those with Human Immune Deficiency Virus infection and hematological disorders are also implicated in increment of pulmonary disease due to NTM (8). Studies done in Canada and US indicate that pulmonary NTM (PNTM) disease is far more common than TB and causes higher morbidity than TB especially in elderly individuals $(12,13)$

Ethiopia is one of the thirty high burden tuberculosis countries in the world (14). According to the 2019 World Health Organization's global TB report, there were an estimated 165,000 incidence cases of tuberculosis (TB,) and the estimated TB cases with MDR/RR-TB in new and previously treated cases were $0.71 \%$ and $16 \%$ respectively (14).

A lot of studies were conducted regarding the burden of Mycobacterium tuberculosis and their drug resistance profile in Ethiopia $(15,16$, $17,18,19,20,21)$. However, in Ethiopia, very few studies report existence of nontuberculous mycobacteria without species identification $(15,20)$. In addition, drug resistance profile and burden of the tuberculosis disease also need to be updated for effective management of tuberculosis patients. Accordingly, this study focused on assessing the magnitude of Mycobacterium tuberculosis and nontuberculous mycobacteria isolates and also determining the drug resistance profile of Mycobacterium tuberculosis isolates from presumptive pulmonary tuberculosis patients attending St. Paul's Hospital Millennium Medical College Addis Ababa, Ethiopia.

\section{MATERIALS AND METHODS}

Study design and Study population: A cross sectional study design was done from June to September 20/2016. Presumptive pulmonary tuberculosis patients who were attending St. Paul's Hospital Millennium Medical College were enrolled to the study. It is the second largest public hospital in the nation which serves more than 110,000 people annually.

Sample size determination and sampling technique: The minimum required sample size of the study was determined using the formula for a single population proportion. By considering prevalence of pulmonary tuberculosis as $21.3 \%$ from previous study done in Addis Ababa, Ethiopia (21), 95\% confidence level and marginal error of 5\%, the final sample size was 258. However, a total of 275 patients with presumptive pulmonary tuberculosis were consecutively included in this study.

Data collection: After informed consent, sociodemographic, health and behavioral factors, clinical history and chest $\mathrm{x}$-ray data were collected from each participants using a structured questionnaire. In addition, about 3-5 $\mathrm{ml}$ of morning sputum samples were collected using sterile, disposable, single-use, screwcapped conical centrifuge tubes. It was 
immediately transported to the national tuberculosis laboratory of Ethiopian public health institute for laboratory processing.

Sample processing and smear microscopy: Sputum samples were mixed with an equal volume of $\mathrm{N}$-acetyl-L-cysteine-sodium hydroxide (NALC-NAOH) solution and vortexed for less than 20 seconds and kept for 15 minutes at $20-25{ }^{\circ} \mathrm{C}$ for the decontamination. Phosphate Buffered saline (PBS) was filled to a $50 \mathrm{ml}$ mark on the falcon tube and vortexed. It was centrifuged at $3000 \mathrm{~g}$ for 15 minutes and the supernatant was discarded. A portion of the sediments was used for culture and the other portion for Ziehl-Neelsen staining after resuspended in $1 \mathrm{ml}$ PBS (22).

Sputum smears of size $1 \times 2 \mathrm{~cm}$ were made with new grease-free slides and allowed to air dry. The air dried smears were then fixed by gently passing over a flame 2-3 times. The smears including positive and negative controls were stained with Ziehl-Neelsen (ZN) technique. Examination and reporting of stained slides were performed by light microscopy (22).

Culture and identification: Portion of each specimen sediment simultaneously inoculated into solid Lowenstein Jensen (2-3 drops) and BACTEC MGIT 960 media (Becton Dickinson, Franklin Lakes, NJ07417, USA) $(0.5 \mathrm{ml})$. LJ medium cultures incubated in a $37^{\circ} \mathrm{C}$ for a maximum of eight weeks (monitored every week) whereas liquid culture was incubated in an automated BACTEC MGIT $960^{\mathrm{TM}}$ machine (Becton Dickinson Diagnostic Instrument Systems) for a maximum of 42 days to report negative. Cultures exhibiting growth were subjected to light microscopy for the presence of acid-fast bacteria and inoculated to blood agar (BAP) before being considered as positive for Mycobacteria (22).

Isolates grown on blood agar after overnight incubation and no acid fast bacilli (AFB) seen on Ziehl-Neelson staining were considered as contaminants. However, isolates which showed no growth on BAP and positive for AFB were considered as Mycobacteria. In addition, isolates grown on BAP and AFB seen on Ziehl-Neelsen staining were considered as the presence of both contaminants and Mycobacteria (22).

Capilia TB-Neo rapid test: Capilia TB-Neo rapid test was used to classify the mycobacteria into Mycobacterium tuberculosis complex (MTBC) and probable Nontuberculous mycobacteria. The Capilia TB-Neo Rapid test negative and positive growth were considered as probable NTM and MTBC respectively. Confirmation and characterization of the probable NTM was performed by Hain"s GenoType ${ }^{\circledR}$ Mycobacterium $\mathrm{CM}$ and GenoType ${ }^{\circledR}$ Mycobacterium AS molecular genetic assays.

$\begin{array}{lcr}\text { DNA extraction } & \text { and } & \text { GenoType } \\ \text { Mycobacterium } & \mathrm{CM} / \mathrm{AS} & \text { assay: }\end{array}$
Nontuberculous DNA was extracted from heatinactivated AFB isolates. Briefly, bacteria suspended in $500 \mu 1$ sterile water or $1 \mathrm{ml}$ directly from positive liquid media were inactivated at $80^{\circ} \mathrm{C}$ for $20 \mathrm{~min}$, then ultrasonicated at $35 \mathrm{kHz}$ and heated at $100{ }^{\circ} \mathrm{C}$ for $10 \mathrm{~min}$ each treatment and centrifuged at 16,100 $\mathrm{g}$ two times for 5 minutes. The supernatant was taken as template DNA (23).

GenoType ${ }^{\circledR} \quad$ Mycobacterium CM/AS (HainLifescience $\mathrm{GmbH}$, Nehren, Germany) assays were performed using the template DNA according to the manufacturer's instructions. The GenoType CM assay was used to identify the common NTM species. GenoType AS assay was used to identify additional NTM species when the species are undetermined by the former assay. Those tests are based on the DNA strip technology. Three controls (conjugate, universal, and genus) are included in each strip (23).

Drug susceptibility test: Phenotypic method using BACTEC-MGIT 960 SIRE Kits (Franklin Lakes, NJ, USA) was done on pure isolates of MTBC. It was performed for the first line antituberculosis drugs using BACTEC MGIT 960 media. The critical concentration of each drug was $1 \mu \mathrm{g} / \mathrm{ml}, 0.1 \mu \mathrm{g} / \mathrm{ml}, 1 \mu \mathrm{g} / \mathrm{ml}, 5 \mu \mathrm{g} / \mathrm{ml}$ and $100 \mu \mathrm{g} / \mathrm{ml}$ for streptomycin, isoniazid, rifampicin, ethambutol and pyrazinamide respectively. The MGIT 960 system 
automatically interpreted the results as susceptible or resistant (22).

Statistical analysis: Data were collected, entered, and analyzed using SPSS version 20 software. Categorical variables were described as proportion. Binary logistic regression was performed to check the presence of an association between MTBC and independent variables. A p-value less than 0.05 was considered as statistically significant.

Ethics approval and consent to participate: Ethical clearance was obtained from the Ethical Review Committee of Medical Laboratory Sciences, College of Health Sciences, Addis Ababa University. Written informed consent was also obtained from all eligible study participants or assent from the guardians of participants younger than 18 years. Laboratory confirmed cases were managed by the clinicians.

\section{RESULTS}

Characteristics of study participants: A total of 275 presumptive pulmonary tuberculosis patients with a predominance of males (150, $54.5 \%)$ were enrolled. The mean age was 43 years ( $\pm 6.6 \mathrm{SD})$ and the majority $(33.1 \%)$ fell in the age range of $31-45$ years (Table 1 ).

Most (96.4\%) of the participants were ambulatory patients. About 36(13.1\%), $75(27.3 \%)$ and $19(6.9 \%)$ of the study participants had HIV infection, history of previous tuberculosis treatment and diabetes mellitus respectively (Table 1).

Clinical features and chest $x$-ray results of study participants: Persistent cough for more than two weeks and chest pain were observed in $259(94.2 \%)$ and $228(82.9 \%)$ participants respectively. Of 224 participants with known chest x-ray results, 111(49.6\%) showed different types of abnormalities (Table 2).
Table 1: Demographic, health and behavioral characteristics of 275 presumptive tuberculosis patients at St. Paul's Hospital Millennium Medical College, 2016.

\begin{tabular}{|c|c|c|}
\hline Variable & Frequency & Percent \\
\hline \multicolumn{3}{|l|}{ Sex } \\
\hline Male & 150 & 54.5 \\
\hline Female & 125 & 45.5 \\
\hline \multicolumn{3}{|l|}{ Residence } \\
\hline Rural & 95 & 34.5 \\
\hline Urban & 180 & 65.5 \\
\hline \multicolumn{3}{|l|}{ Age } \\
\hline $16-30$ & 78 & 28.4 \\
\hline $31-45$ & 91 & 33.1 \\
\hline $46-60$ & 62 & 22.5 \\
\hline$\geq 61$ & 44 & 16 \\
\hline \multicolumn{3}{|l|}{ Educational level } \\
\hline Illiterate & 102 & 37.1 \\
\hline$<6$ & 42 & 15.3 \\
\hline $6-12$ & 105 & 38.2 \\
\hline Higher education & 26 & 9.5 \\
\hline \multicolumn{3}{|l|}{ Occupation } \\
\hline Farming & 84 & 30.5 \\
\hline Office work/student & 69 & 25.1 \\
\hline House wife & 75 & 27.3 \\
\hline Others & 47 & 17.1 \\
\hline \multicolumn{3}{|l|}{ Previous TB treatment } \\
\hline Yes & 75 & 27.3 \\
\hline No & 200 & 72.7 \\
\hline \multicolumn{3}{|l|}{ HIV status } \\
\hline Reactive & 36 & 13.1 \\
\hline Non-reactive & 77 & 28 \\
\hline Unknown & 162 & 58.9 \\
\hline \multicolumn{3}{|l|}{ Diabetes mellitus } \\
\hline Yes & 19 & 6.9 \\
\hline No & 54 & 19.6 \\
\hline Unknown & 202 & 73.5 \\
\hline \multicolumn{3}{|l|}{ Smoke cigarette } \\
\hline Yes & 16 & 5.8 \\
\hline No & 259 & 94.2 \\
\hline \multicolumn{3}{|l|}{ Drink alcohol } \\
\hline Yes & 58 & 21.1 \\
\hline No & 217 & 78.9 \\
\hline \multicolumn{3}{|l|}{ Chew Kchat } \\
\hline Yes & 21 & 7.6 \\
\hline No & 254 & 92.4 \\
\hline
\end{tabular}


Table 2: Distribution of clinical and chest x-ray results of 275 presumptive tuberculosis patients at St. Paulo's hospital millennium medical college, 2016.

\begin{tabular}{lll}
\hline Variable & Frequency & Percent \\
\hline Cough $\geq 2$ weeks & & \\
$\quad$ Yes & 259 & 94.2 \\
$\quad$ No & 16 & 5.8 \\
Hemoptysis & & \\
$\quad$ Yes & 77 & 28 \\
$\quad$ No & 198 & 72 \\
Night sweeting & & \\
$\quad$ Yes & 151 & 54.9 \\
$\quad$ No & 124 & 45.1 \\
Fever & & \\
Yes & 182 & 66.2 \\
$\quad$ No & 93 & 33.8 \\
Chest pain & & \\
Yes & 228 & 82.9 \\
$\quad$ No & 47 & 17.1 \\
Chest x-ray result & & \\
$\quad$ Normal & 113 & 41.1 \\
Abnormal & 111 & 40.4 \\
$\quad$ Unknown & 51 & 18.5 \\
\hline
\end{tabular}

Magnitude of mycobacterium tuberculosis complex and nontuberculous mycobacteria:

Twenty-nine $(10.6 \%, 29 / 275)$ morning sputum samples were found to be culture positive for Mycobacteria. Of the 29 participants with confirmed mycobacteria, 26(89.7\%) were MTBC and the remaining 3(10.3\%) were NTM. Sputum smear microscopy identified TB in 12 $(45.2 \%)$ cases. These resulted in an overall magnitude of $9.5 \%(26 / 275)$ for MTBC and $1.1 \%(3 / 275)$ for NTM. There was no coisolation of MTBC and NTM in the sputum samples examined.

Of the three isolated nontuberculous mycobacteria, two were unidentified NTM and one was identified as Mycobacterium peregrinum. Two of the isolated NTM were rapid growers. All the NTM were negative for direct sputum smear microscopy.

Mycobacterium tuberculosis magnitude and chest $x$-ray results: We assessed the chest $x$-ray profiles of 224 study subjects out of 275 patients. The chest x-ray results of 51 patients $(18.5 \%, 51 / 275)$ were not available during data mining from the patient card. The radiologist reported normal chest x-ray results for 113 subjects (113/ 224). However, 8 patients $(7.1 \%$, $8 / 113)$ were positive for MTBC using culture (Table 3).

Regarding nontuberculous mycobacteria, two were from patients who had normal chest $\mathrm{x}$ ray results and one from a patient who had abnormal chest $\mathrm{x}$-ray result.

Table 3: Frequency of Mycobacterium tuberculosis complex isolates along with chest x-ray results at St. Paul's Hospital Millennium Medical College, 2016.

\begin{tabular}{cccccc}
\hline Chest x-ray result & Total & $\begin{array}{c}\text { MTBC Isolate } \\
\text { Number(\%) }\end{array}$ & $\begin{array}{c}\text { Smear } \\
\text { positive }\end{array}$ & $\begin{array}{c}\text { Smear negative/ culture } \\
\text { positive }\end{array}$ \\
\hline Normal & 113 & $8(32 \%)$ & 2 & 6 \\
Abnormal & 111 & $17(68 \%)$ & 10 & 7 \\
& Total & 224 & $25(100)$ & 12 & 13 \\
\hline
\end{tabular}

MTBC- Mycobacterium tuberculosis complex

Table 4: Distribution of drug resistance pattern of 26 isolated Mycobacterium tuberculosis from patients at St. Paulo's hospital millennium medical college, 2016.

\begin{tabular}{lc}
\multicolumn{1}{c}{ Sensitivity/Resistance } & Number (\%) \\
\hline Sensitive to all drugs & $20(76.9 \%)$ \\
Single resistance & \\
$\quad$ Streptomycin & $3(11.5 \%)$ \\
$\quad$ Isoniazid & $1(3.8 \%)$ \\
Double resistance & \\
$\quad$ Rifampicin and Isoniazid & $1(3.8 \%)$ \\
Triple resistance & \\
$\quad$ Rifampicin, Isoniazid, Streptomycin & $1(3.8 \%)$ \\
\hline
\end{tabular}


Drug resistant profile of Mycobacterium tuberculosis complex isolates: Of the twenty six isolated Mycobacterium tuberculosis complex, $6(23.1 \%)$ isolates were resistant to at least one drug. The highest proportions of any drug resistance were observed in Streptomycin 4(15.4 $\%$ ) followed by Isoniazid 3(11.5\%). Two MDR cases $(7.7 \%)$ were isolated from patients who had a previous tuberculosis treatment history (Table 4).
Mycobacterium tuberculosis complex (MTBC) and associated factors: Larger proportion of females $(9.6 \%, p=0.940)$, participants within the age range of $16-30$ years $(14.1 \%, p=0.119)$ and HIV infected patients $(8.3 \%, \mathrm{p}=0.723)$ were TB positive. The pulmonary TB positive and negative individuals had no significant differences concerning educational status, occupation, residence, marital status, HIV status, DM status, alcohol consumption, and smoking cigarette (Table 5).

Table 5: Association between socio-demographic, behavioral, clinical characteristics and MTBC positivity of study participants at St. Paul's Hospital Millennium Medical College, 2016.

\begin{tabular}{|c|c|c|c|c|}
\hline Variable & Positive N (\%) & COR (CI; 95\%) & P-value & \\
\hline \multicolumn{5}{|l|}{ Sex } \\
\hline Male & $14(53.8)$ & $1.119(0.443-2.824)$ & 0.940 & \\
\hline Female & $12(46.2)$ & I & & \\
\hline Residence & & & & V0.393 \\
\hline Rural & $19(73.1)$ & $1.484(0.6-3.666)$ & 0.393 & \\
\hline Urban & $7(26.9)$ & I & & \\
\hline \multicolumn{5}{|l|}{ Age } \\
\hline $16-30$ & $11(42.3)$ & $0.290(0.061-1.374)$ & 0.119 & \\
\hline $31-45$ & $9(34.6)$ & $0.434(0.090-2.099)$ & 0.299 & \\
\hline $46-60$ & $4(15.4)$ & $0.690(0.121-3.947)$ & 0.677 & \\
\hline$\geq 61$ & $2(7.7)$ & $\mathbf{I}$ & & 0.984 \\
\hline \multicolumn{5}{|l|}{ Educational level } \\
\hline Illiterate & $5(19.2)$ & $2.53(0.564-11.36)$ & 0.226 & \\
\hline$<6$ & $5(19.2)$ & $0.965(0.210-4.427)$ & 0.964 & \\
\hline $6-12$ & $13(50)$ & $0.923(0.243-3.511)$ & 0.907 & 0.9070 .907 \\
\hline Higher education & $3(10.3)$ & I & & \\
\hline \multicolumn{5}{|l|}{ Occupation } \\
\hline Farming & $8(30.8)$ & $0.884(0.251-3.107)$ & 0.847 & \\
\hline Office work/student & $7(26.9)$ & $0.904(0.259-3.271)$ & 0.904 & \\
\hline House wife & $7(26.9)$ & $0.824(0.227-2.989)$ & 0.824 & \\
\hline Others & $4(11.515 .4)$ & $\mathbf{I}$ & & \\
\hline Previous TB treatment & & & & 00 \\
\hline Yes & $5(19.2)$ & $1.642(0.596-4.526)$ & 0.337 & \\
\hline No & $21(80.8)$ & I & & \\
\hline \multicolumn{5}{|l|}{ HIV status } \\
\hline Reactive & $3(11.5)$ & $0.764(0.172-3.388)$ & 0.723 & \\
\hline Non-reactive & $5(17.2)$ & I & & \\
\hline \multicolumn{5}{|l|}{ Diabetes mellitus } \\
\hline Yes & $2(7.7)$ & $1.70(0.333-8.682)$ & 0.521 & \\
\hline No & $9(34.6)$ & I & & \\
\hline \multicolumn{5}{|l|}{ Smoke cigarette } \\
\hline Yes & $2(7.7)$ & $0.715(0.153-3.335)$ & 0.669 & \\
\hline No & $24(92.3)$ & I & & \\
\hline \multicolumn{5}{|l|}{ Drink alcohol } \\
\hline Yes & $7(24.1)$ & $0.699(0.279-1.754)$ & 0.446 & \\
\hline No & 19(75.9) & I & & \\
\hline
\end{tabular}

I-Reference category, COR- Crude Odds Ratio, CI-Confidence interval, N-Number, TB-Tuberculosis 


\section{DISCUSSION}

Isolation and detection of mycobacteria play an important role in the control and prevention of tuberculosis and tuberculosis like diseases (24). During the four month period of our study, a total of 275 presumptive pulmonary TB patients were enrolled, and $29(10.6 \%)$ of them were positive for Mycobacteria infection. Of these, the majority were MTBC 26(89.7\%). M.peregrinum was the NTM species identified.

A similar study on identification of mycobacteria in Nigeria reported that out of 270 suspected pulmonary tuberculosis patients, 26(9.5\%) were infected with mycobacteria (25), which is comparable to our finding. However, higher isolation rates were reported from Tanzania, (32.5\%) (26) and Ethiopia, (17.4\%) (15).

The overall magnitude of bacteriologically confirmed pulmonary tuberculosis in our study $(9.5 \%)$ was lower than previous reports in Ethiopia (16.5\%) (16), Tanzania (22.8\%) (26) and Nigeria (28\%) (27). However, our finding showed a higher burden than another study conducted in Nigeria (6.3\%) (25). These may reflect the variations in the study population and laboratory method. Of the cultured confirmed TB, $32 \%(8 / 25)$ patients had normal chest $\mathrm{x}$-ray results. This implies that the use of sputum culture in the diagnosis of active tuberculosis improves detection rates and ultimately leads to improved patient outcomes and reduced transmission of $M$. tuberculosis.

Previous reports in Ethiopia from National Tuberculosis Reference Laboratory (6\%) and Saint Peter Tuberculosis Specialized Hospital Laboratory $(7 \%)$ indicated presence of NTM without confirmation and speciation $(15,20)$. The present study unlike the former revealed higher percentage $(10.3 \%)$ of confirmed nontuberculous mycobacteria. This finding is consistent with studies done in Iran (10.2\%) (3) and Tanzania (9.7\%) (26), but higher isolation rate was observed in USA (14\%) (14) and Nigeria (15\%) (27). This might be due to the variation in study population and laboratory method.
The distribution of NTM species significantly varies across regions (10). Of the 3 NTM isolated in this study, molecular technique showed that one NTM was typed as $M$. peregrinum. Studies carried out in some African countries identified $M$. peregrinum and other NTM species from sputum samples of presumptive pulmonary tuberculosis patients $(28,29,8)$. Additionally, $M$. peregrinum implicated as a possible causative agent of pulmonary disease in human $(30,31)$. However, the NTM isolates in this study were not evaluated by the diagnostic criteria for NTM disease published by the American Thoracic Society (ATS).

In this study, a resistance proportion to at least one of the five anti-tuberculosis drug tested was $23.1 \%$ which is comparable to reports in Addis Ababa, Ethiopia (22.3\%) (17) and Libya (24.1\%) (32). However, higher resistance proportion was reported in other studies done in Ethiopia (27.4\%) (18) and Kenya (30.1\%) (33). This variation could be due to the fact that the latter two studies were conducted at referral sites for TB cases. A study done in East Gojam, Ethiopia, reported slightly lower level (20.23\%) of drug resistance than our study (19).

This study has some limitations. There was no follow-up of patients who were positive for nontuberculous mycobacteria even though reported to the concerned physician. The study was also limited to patients who were attended to in one hospital, but the information reported in this study could be a significant contribution to the existing knowledge on the burden of MTBC and nontuberculous mycobacteria.

In conclusion, this study depicted that the majority of the isolated Mycobacteria from sputum samples of presumptive pulmonary tuberculosis patients were Mycobacterium tuberculosis complex (89.7\%) and low extent of nontuberculous mycobacteria $(10.3 \%)$. Of the isolated MTBC, $76.9 \%$ were susceptible to five anti-tuberculosis drugs. Therefore, further large scale study is needed to substantiate those results and to consider NTM as a differential diagnosis of pulmonary tuberculosis like disease. 


\section{ACKNOWLEDGMENT}

Our sincere gratitude goes to Addis Ababa University and Ethiopian Public Health Institute, especially National Tuberculosis Reference Laboratory staff. All laboratory staff of St. Paul's Hospital Millennium Medical College should be acknowledged. Finally, we would like to thank the study participants for their willingness to give their consent to be included in this study.

\section{REFERENCES}

1. Panagiotou M, Papaioannou AI, Kostikas K, et al. The Epidemiology of Pulmonary Nontuberculous Mycobacteria: Data from a General Hospital in Athens, Greece, 20072013. Pulmonary Medicine. 2014; 2014. doi: 10.1155/2014/894976.

2. Kendall BA, Varley CD, Choi D, et al. Distinguishing tuberculosis from nontuberculous mycobacteria lung disease, Oregon, USA. Emerging Infectious Diseases.2011; 17:3:506-509.

3. Nasiri MJ, Dabiri H, Darban-Sarokhalil D, Hashemi-Shahraki A. Prevalence of nontuberculosis Mycobacterial Infections among Tuberculosis Suspects in Iran: Systematic Review and Meta-Analysis. PLoS One. 2015; 10:6. e0129073.

4. Pokam BT, Asuquo AE. Acid-Fast Bacilli Other than Mycobacteria in Tuberculosis Patients Receiving Directly Observed Therapy Short Course in Cross River State, Nigeria. Tuberculosis Research and Treatment. 2012:2012. doi:10.1155/2012/301056.

5. World Health Organization: WHO Report 2016. Global tuberculosis control 2016 Geneva: World Health Organization; 2016.

6. Asiimwe BB, Bagyenzi GB, Ssengooba W, et al. Species and genotypic diversity of non-tuberculous mycobacteria isolated from children investigated for pulmonary tuberculosis in rural Uganda. BMC Infectious Diseases. 2013; 13(1):88.

7. $\mathrm{Wu} \mathrm{T}, \mathrm{Lu} \mathrm{C}$, Lai H. Current situations on identification of Nontuberculous mycobacteria. Journal of Biomedical Laboratory Science.2009; 21(1):1-6.
8. Kendall BA, Varley CD, Hedberg K, Cassidy PM, Winthrop KL. Isolation of nontuberculous mycobacteria from the sputum of patients with active tuberculosis. International Journal of Tuberculosis and Lung Disease. 2010; 14(5):654-656.

9. Satta G, McHugh TD, Mountford J, Abubakar I, Lipman M. Managing pulmonary nontuberculous mycobacterial infection. Time for a patient-centered approach. Annals of the American Thoracic Society. 2014; 11(1):117-21.

10. Kendall BA, Winthrop KL. Update on the epidemiology of pulmonary nontuberculous mycobacterial infections. Seminars in Respiratory and Critical Care Medicine. 2013; 34(1):87-94. doi: 10.1055/s-00331333567.

11. Rivero-Lezcano OM, González-Cortés C, Mirsaeidi M. The unexplained increase of nontuberculous mycobacteriosis. International Journal of Mycobacteriology. 2019; 8(1):1.

12. Damaraju D, Jamieson F, Chedore P, Marras TK. Isolation of non-tuberculous mycobacteria among patients with pulmonary tuberculosis in Ontario, Canada. International Journal of Tuberculosis and Lung Disease.2013; 17 (5): 676-681.

13. Mirsaeidi M, Farshidpour M, Allen MB, Ebrahimi G, Falkinham JO. Highlight on Advances in Nontuberculous Mycobacterial Disease in North America. 2014; 2014.

14. World Health Organization: WHO Report 2019 for 30 high-burden countries profile. Geneva: World Health Organization; 2019.

15. Desta K, Asrat D, Lemma E, Gebeyehu M, Feleke B. Prevalence of smear negative pulmonary tuberculosis among patients visiting St. Peter's Tuberculosis Specialized Hospital, Addis Ababa, Ethiopia. Ethiopian Medical Journal. 2009; 47(1):17-24.

16. Worku M, Befikadu T. Prevalence of rifampicin mono resistant Mycobacterium tuberculosis among Suspected Cases Attending at Yirgalem Hospital. Clinical Medicine Research. 2015; 4(3): 75-78.

17. Eyob G, Gebrexabher H, Lemma E, et al. Drug susceptibility of Mycobacterium tuberculosis in HIV-infected and uninfected 
Ethiopians and its impact on outcome after 24 months of follow-up. International Journal of Tuberculosis and Lung Disease. 2004; 8(11):1388-1391.

18. Desta K, Asrat D, Lemma E, Gebeyehu M, Feleke B. Drug susceptibility of Mycobacterium tuberculosis isolates from smear negative pulmonary tuberculosis patients, Addis Ababa, Ethiopia. Ethiopian journal of Health Development. 2008; 22(2):212-215.

19. Adane K, Ameni G, Bekele S, Abebe M, Aseffa A. Prevalence and drug resistance profile of Mycobacterium tuberculosis isolated from pulmonary tuberculosis patients attending two public hospitals in East Gojjam zone, northwest Ethiopia. BMC Public Health. 2015; 15:572.

20. Mathewos B, Kebede N, Kassa T, Mihret A, Getahun M. Characterization of mycobacterium isolates from pulmonary tuberculosis suspected cases visiting Tuberculosis Reference Laboratory at Ethiopian Health and Nutrition Research Institute, Addis Ababa Ethiopia: a cross sectional study. Asian Pacific Journal of Tropical Medicine. 2015; 35-40.

21. Deribew A, Negussu N, Melaku Z, Deribe $\mathrm{K}$. Investigation Outcomes of Tuberculosis Suspects in the Health Centers of Addis Ababa, Ethiopia. PLoS ONE. 2011; 6 (4):e18614.

22. Mycobacteriology laboratory manual. A publication of the Global Laboratory Initiative a Working Group of the Stop TB Partnership. First Edition, April 2014. Available at: www.who.int/tb/laboratory/mycobacteriolog y-laboratory-manual.pdf

23. Geno Type Mycobacterium CM/AS assay manual. 2016. Available at: https://www.immunodiagnostic.fi/wpcontent/uploads/GenoType-CM-V2 kitinsert.pdf

24. Shrestha NK, Tuohy MJ, Hall GS, Reischl U, Gordon SM, Procop GW. Detection and Differentiation of Mycobacterium tuberculosis Nontuberculous Mycobacterial Isolates by Real-Time PCR. Journal of clinical microbiology. 2003; 41 (11): 51215126.

25. Jimoh O, Olayinka A, Musa BO, Ezati N, Ajiboye PF. Prevalence of culture-positive mycobacteria among suspected cases of pulmonary tuberculosis in Ahmadu Bello university teaching hospital, Zaria, Northern, Nigeria. International Journal of Infectious Diseases. 2016:45:1-477. doi: 10.1016/j. ijid. 2016.02.847.

26. Hoza AS, Mfinanga SG, Rodloff AC, Moser I, König B. Increased isolation of nontuberculous mycobacteria among TB suspects in Northeastern, Tanzania: public health and diagnostic implications for control programs. BMC Res Notes. 2016; 9(109):1928-3.

27. Aliyu G, El-Kamary SS, Abimiku A, et al. Prevalence of non-tuberculous mycobacterial infections among tuberculosis suspects in Nigeria. PLoS One. 2013; 8 (5): e63170.

28. Nyamogoba H DN, Mbuthia G, Mining S, et al. HIV coinfection with tuberculous and non-tuberculous mycobacteria in western Kenya: challenges in the diagnosis and management. African Health Sciences. 2012; 12(3):305-311.

29. Bjerrum S, Oliver-Commey J, Kenu E, Lartey M, Newman MJ, Addo KK, et al. Tuberculosis and non-tuberculous mycobacteria among HIV infected individuals in Ghana. Tropical Medicine and International Health.2016; 21(9):1181 1190.

30. Sawahata M, Hagiwara E, Ogura T, et al. Pulmonary mycobacteriosis caused by Mycobacterium peregrinum in a young, healthy man. J of IMAB. 2015:21(4).

31. Todorova T, Kaludova V, Tsankova G, Ermenlieva N. A pulmonary infection caused byMycobacterium peregrinum - a case report. Nihon Kokyuki Gakkai Zasshi. 2010; 48(11):866-70.

32. Albarouni AS, Aboguttaia NB, Alamri WM, Fungi AM, Furjani M, Zorgani A. Drug Susceptibility Patterns of Mycobacterium tuberculosis Isolated from Patients with Pulmonary Tuberculosis in Tripoli-Libya. 
Journal of Mycobacterial Disease. 2014; 4:3.

33. Ndungee $\mathrm{P}$ PW, Kariuki S, Ngeeangee a Z, Revathi
G. Resistance patterns of
Mycobacterium tuberculosis isolates from pulmonary tuberculosis patients in Nairobi. Journal of Infection in Developing Countries. 2012; 6(1):33-39. 